\title{
Radiographic diagnosis of a respiratory condition mimicking tension pneumothorax
}

\author{
HIROYUKI YAMAGUCHI ${ }^{1}$ and Kazuya Nagasaki ${ }^{1}$
}

${ }^{1}$ Mito Kyodo General Hospital

September 14, 2020

\begin{abstract}
Massive empyema can mimic tension pneumothorax clinically and can be distinguished only radiographically. This distinction is crucial since needle decompression for tension pneumothorax can cause iatrogenic pneumothorax in those with massive empyema.
\end{abstract}

Title

Radiographic diagnosis of a respiratory condition mimicking tension pneumothorax

Authors

Hiroyuki Yamaguchi ${ }^{1}, \mathrm{MD}$; Kazuya Nagasaki ${ }^{1}$, MD

Author Affiliations

${ }^{1}$ Department of Internal Medicine, Mito Kyodo General Hospital, University of Tsukuba, Japan

Corresponding Author's Information

Name: Hiroyuki Yamaguchi

Contact Information (e-mail):hiy.yamaguchi@gmail.com

\section{Key Clinical Message}

Massive empyema can mimic tension pneumothorax clinically and can be distinguished only radiographically. This distinction is crucial since needle decompression for tension pneumothorax can cause iatrogenic pneumothorax in those with massive empyema.

\section{Clinical image}

A 67-year-old male smoker presented with a 3-day history of severe progressive acute respiratory distress (pulse, 120 beats/min, respiration, 40 breaths/min, and oxygen saturation, $72 \%$ ). Physical examination revealed left tracheal deviation, decreased right chest wall movement, and diminished breath sounds. We suspected tension pneumothorax and scheduled an emergency needle decompression. Pre-procedural lung ultrasound showed fluid accumulation in the right chest; chest radiography revealed large pleural effusion without pneumothorax (Fig. 1a); and chest computed tomography showed massive, loculated pleural effusion with gas (Fig. 1b). What is your diagnosis?

\section{Discussion}

Thoracic drainage through a chest tube confirmed empyema. Tachycardia and hypoxia improved with drainage of two liters of pus.Streptococcus intermedius was detected in the pleural fluid and blood cultures. 
After 4 weeks of antibiotic therapy with 15 days of thoracic drainage, the patient was discharged without complications.

Massive empyema can cause a mediastinal shift, diminished breath sounds, and respiratory distress, mimicking tension pneumothorax. ${ }^{1}$ Lung ultrasound and chest radiography can help differentiate between both. ${ }^{2}$ Emergency needle decompression at the second intercostal space is recommended for tension pneumothorax; however, this can cause iatrogenic pneumothorax in patients with massive empyema. (Fig. 1a: arrow). Therefore, chest imaging should be performed before such procedures.

\section{Patient Consent}

Written informed consent was obtained from the patient.

\section{Funding}

None

\section{Conflict of Interest}

None declared.

\section{Author contribution}

Hiroyuki Yamaguchi - Writing (Original Draft)

Kazuya Nagasaki - Writing (Review \& Editing)

\section{References}

1) Baker L, Carlson R. Streptococcus acidominimus isolated from a multiloculated empyema in a critically ill adult man with pneumonia: case report and review of literature. Heart Lung 2008; 37:308-10.

2) Nelson M, Stankard B, Greco J, Okumura Y. Point of Care Ultrasound Diagnosis of Empyema. J Emerg Med 2016; 51:140-3.

\section{Figure Legends}

Fig. 1a: Chest radiography (dorsal) showing a large pleural effusion without any findings of pneumothorax. Arrow: Empyema misdiagnosed as tension pneumothorax would have punctured the second intercostal space.

Fig. 1b: Trunk computed tomography (coronal) showing pleural effusion on the right side. 


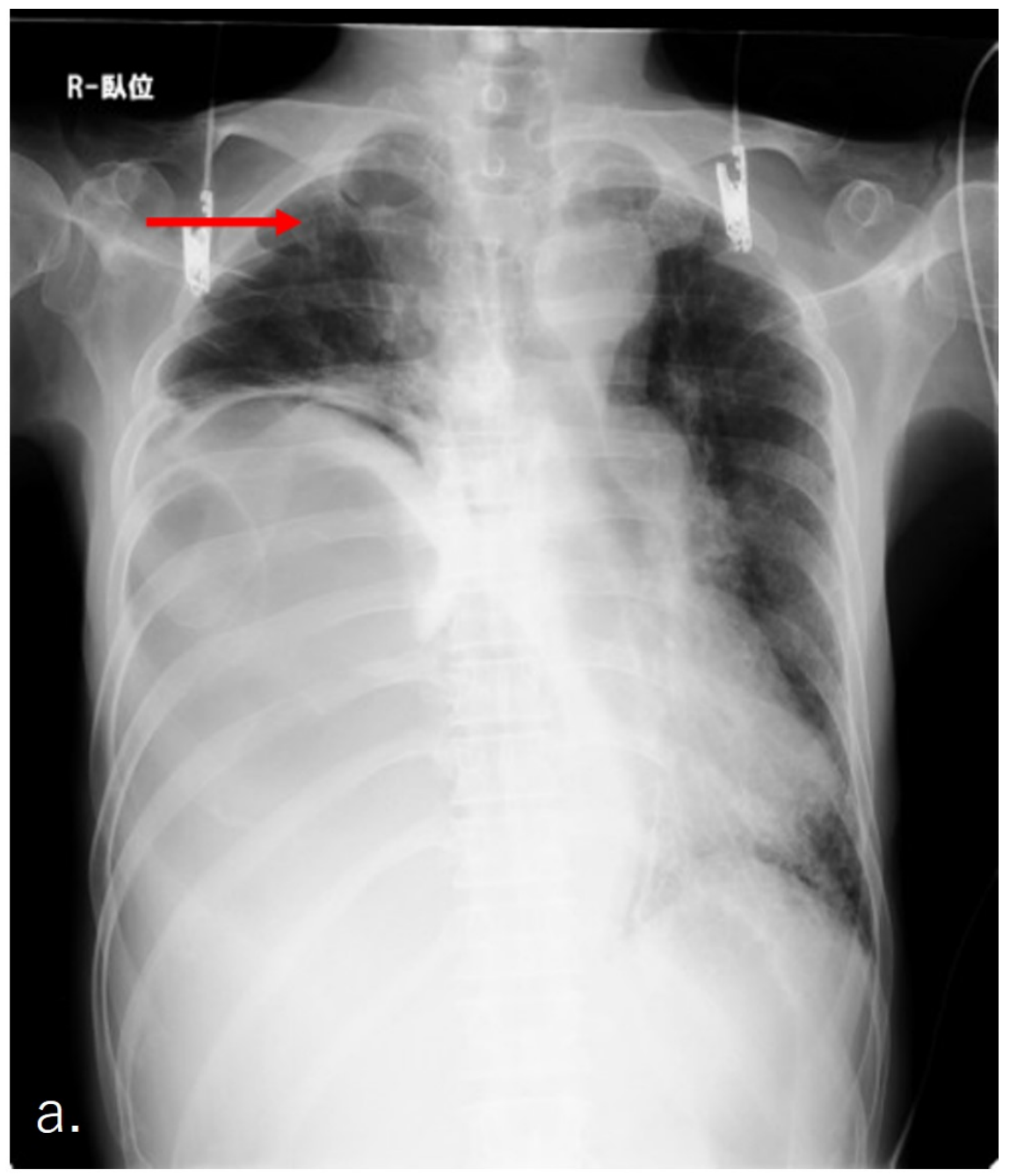




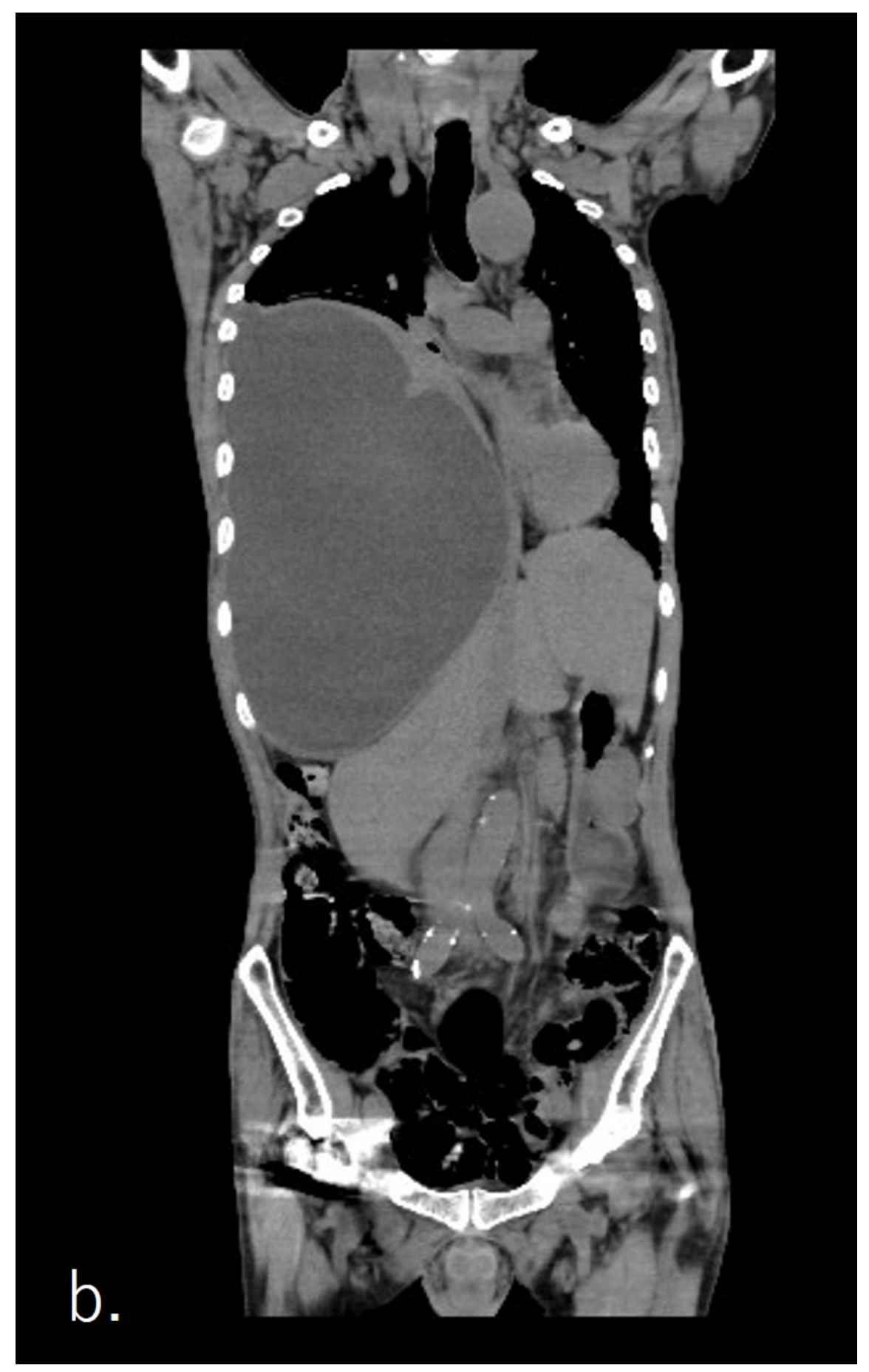

